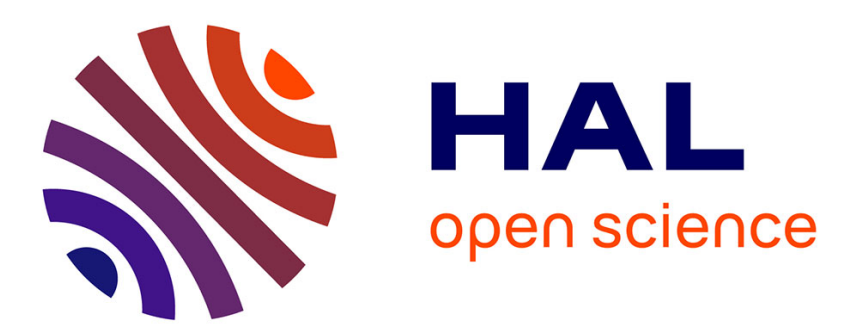

\title{
Decision-Support Methodology to Assess Risk in End-of-Life Management of Complex Systems
}

Eric Villeneuve, Cédrick Béler, François Pérès, Laurent Geneste, Eric Reubrez

\section{To cite this version:}

Eric Villeneuve, Cédrick Béler, François Pérès, Laurent Geneste, Eric Reubrez. Decision-Support Methodology to Assess Risk in End-of-Life Management of Complex Systems. IEEE Systems Journal, 2017, 11 (3), pp.1579-1588. 10.1109/JSYST.2016.2522183 . hal-02319769

\section{HAL Id: hal-02319769 \\ https://hal.science/hal-02319769}

Submitted on 18 Oct 2019

HAL is a multi-disciplinary open access archive for the deposit and dissemination of scientific research documents, whether they are published or not. The documents may come from teaching and research institutions in France or abroad, or from public or private research centers.
L'archive ouverte pluridisciplinaire $\mathbf{H A L}$, est destinée au dépôt et à la diffusion de documents scientifiques de niveau recherche, publiés ou non, émanant des établissements d'enseignement et de recherche français ou étrangers, des laboratoires publics ou privés. 


\title{
Decision-Support Methodology to Assess Risk in End-of-Life Management of Complex Systems
}

\author{
Éric Villeneuve, Cédrick Béler, François Pérès, Laurent Geneste, and Eric Reubrez
}

\begin{abstract}
End-of-life management of complex systems is increasingly important for industry because of growing environmental concerns and associated regulations. In many areas, lack of hindsight and significant statistical information restricts the efficiency of end-of-life management processes and additional expert knowledge is required. In this context and to promote the reuse of secondhand components, a methodology supported by risk assessment tools is proposed. The proposal consists of an approach to combine expert and statistical knowledge to improve risk assessment. The theory of belief functions provides a common framework to facilitate fusion of multisource knowledge, and a directed evidential network is used to compute a measure of the risk level. An additional indicator is proposed to determine the result quality. Finally, the approach is applied to a scenario in aircraft deconstruction. In order to support the scientific contribution, a software prototype has been developed and used to illustrate the processing of directed evidential networks.
\end{abstract}

Index Terms-Belief functions, decision-support system, directed evidential networks, end-of-life management, risk assessment.

\section{INTRODUCTION}

$\mathbf{E}$ ND-OF-LIFE aircraft deconstruction has been rapidly increasing for several reasons. First, there has been a substantial increase in the the number of aircraft that have reached their end-of-life in aircraft fleet. In addition, recent regulatory changes have significantly increased the manufacturer liability regarding the management of end-of-life of their aircraft. Finally, current environmental concerns encourage aircraft manufacturers to include an environmental dimension in their industrial strategies.

The study, presented in this article, was realized as part of the "DIAGNOSTAT" project. ${ }^{1}$ This project focuses on the recovery and reuse of parts as spares.

Certification is costly and it is therefore important to determine as soon as possible the suitability of deconstruction and certification of a part by limiting the number of "physical" examinations required. The objective of this work is to propose mechanisms to evaluate part status at a reduced cost by assessing, a priori, the risk that a part may have defects. Two information sources may provide knowledge to the model: expert opinions and statistical data. The heterogeneity of these

The authors are with the Laboratoire Génie de Production (LGP), Université de Toulouse-école Nationale d'Ingénieurs de Tarbes (ENIT), 65000 Tarbes, France (e-mail: eric.villeneuve@enit.fr; cedrick.beler@enit.fr; francois.peres@ enit.fr; laurent.geneste@enit.fr; eric.reubrez@enit.fr).

\footnotetext{
${ }^{1}$ Project supported by the Aerospace Valley cluster and funded by the French "fond unique interministériel" (FUI).
}

information sources implies a need for hybridization of different knowledge types. Hence, it is essential that uncertainty in this process be taken into account for a coherent risk assessment. In this context and to enable the reuse of secondhand components, a methodology supported by risk assessment tools is proposed and consists of an approach to combine expert and statistical knowledge to improve risk assessment.

A generic risk assessment model was proposed based on a literature review [1]. This model is based mainly on influence diagrams and provides a risk assessment for a part or subassembly of a system. Bayesian networks [2] and their extension, influence diagrams, were used since they are able to model a risk situation simply.

However, uncertainty modeling is complex because of the limitations set by Bayesian formalism and specifically by the underlying probabilistic mechanisms. Hence, the goal is to model information that reflects expertise. Although probability theory, which is used by the Bayesian network formalism, can be used to quantify the randomness of information (variability), it does not allow for easy integration of epistemic information (incompleteness, imprecision). The symmetry or indifference principle (equiprobability) does not distinguish between the representation of a random situation and a partially described context [3]. Within this framework, belief functions can be of a great help. Belief functions are used to merge and integrate this knowledge into the model. Consequently, their integration into a graphical model similar to Bayesian network models has been considered, and thus directed evidential networks are used to perform a risk assessment by taking into account epistemic uncertainty.

This paper introduces the proposed risk assessment methodology. The different phases of this process (knowledge formalization, information fusion, processing into the model, and resulting indicators) are then described. Finally, current work concerning an application of the risk assessment process to aircraft deconstruction is presented.

\section{RISK ASSESSMENT METHOdOLOGY}

\section{A. Risk}

Literature contains many definitions of the risk concept [4]. These definitions often present risk as a combination of hazard and vulnerability. The chosen definition is the one proposed in [5], which defines risk as an event that occurs as an association between "cause events" that are characterized by their occurrence (P), and "consequence events" (effects) that are characterized by their severity or impact (I). Thus, risk can be determined from $R=f$ (occurrence, impact) $=f(P, I)$. 


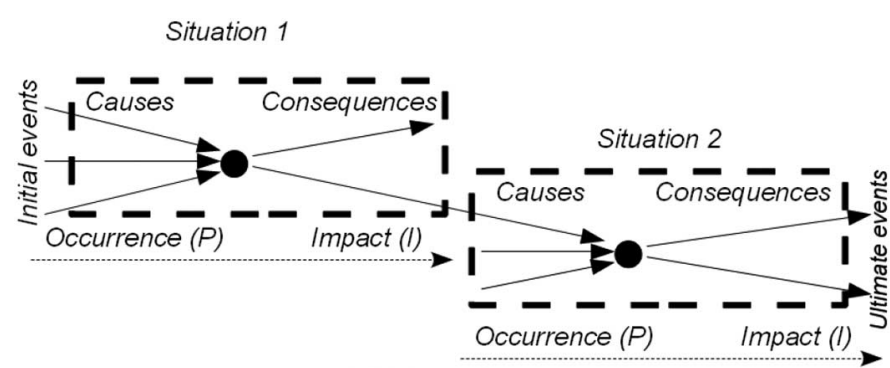

Fig. 1. Representation of the risk sequence.

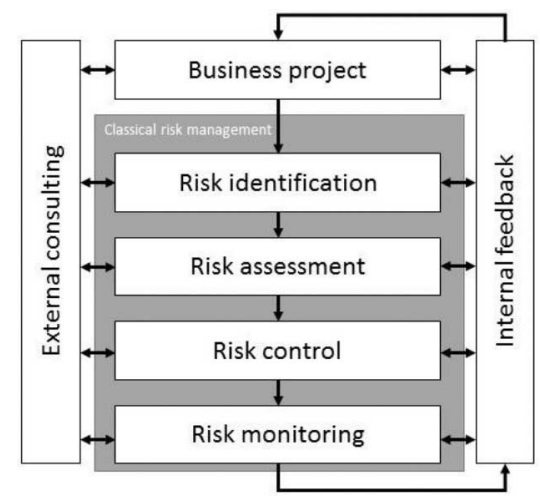

Fig. 2. Organization of the ERM subprocesses based on [8] and [9].

Traditionally, risk is defined by the relationship $R=P \times I$, but literature contains many other formulations. For example, the formula $R=P \times I^{k}[6]$ may be used to highlight risks with high impact because of the coefficient $k$.

This vision can easily be extended to more complex risks by using an event sequence (Fig. 1) where each risk situation is composed of "cause events," "consequence events," and a "target event." It is possible to observe a chain of situations (a "cause event" of a situation is a "consequence event" of another, and vice versa) to study the root causes or the ultimate consequences.

\section{B. Enterprise Risk Management (ERM)}

ERM aims to facilitate the definition of an overall strategy for an organization by identifying events that could affect it, and by controlling these risks to provide reasonable assurance regarding the objectives of this organization [7]. Fig. 2 introduces the various phases of this process.

Many tools and methodologies have been developed in recent years to instrument ERM subprocesses such as [10], [11] for the identification phase, [12] for the control phase, [13], [14] for the monitoring phase, or [5] for the feedback phase. The work described in this paper takes place in the assessment phase of ERM. This phase is usually carried out either by statistical data analysis (quantitative approach) or by interviewing one or more experts (qualitative approach). The proposed approach allows one to assess the risk by following a dedicated methodology which includes hybridization of expert knowledge and statistical data and original measures of risk and uncertainty. The objective is to obtain a quantitative analysis supported by expertise in cases where the amount of data is insufficient for reliable statistics. The proposed process is described in the next section.

\section{Risk Assessment Process}

The network structure, i.e., the event chain representing the various studied risks (see Section II-A), is considered as known and has been defined by experts in the field who identified different possible defects with their associated causes and consequences. This definition is the starting point for building the network structure with arcs and nodes for a given system. All nodes are also considered as discrete variables.

To edit and process expert and statistical knowledge, to assess risk and communicate results via indicators, the proposed process is divided into four successive phases.

1) The first phase, termed "knowledge formalization," consists of expert opinion elicitation and statistical knowledge extraction, both of which result in belief functions.

2) In the second phase, termed "information fusion," one single belief function is obtained for a given set of information (out of several potential sets). This information may consist of several expert opinions and data records. This resulting belief function is subsequently introduced to the corresponding node of the network.

3) The third phase, termed "information processing," is an introduction of any new piece of information and its propagation (evidential inference algorithms) throughout the network nodes to update risk measures.

4) The last phase, termed "result restitution," consists of an interpretation of the final network state to provide the final user with some understandable indicators.

As opposed to the usage of Bayesian networks, the relevance of the chosen approach has increased because the use of belief functions enables the representation of different information sources. As mentioned previously, processing of belief functions correctly takes into account the epistemic uncertainty that is inherent in expert opinions as in sparse statistical data.

The following synoptic view (Fig. 3) is introduced to describe further the required sequence of actions for this transformation. It consists of a two-headed process with expert opinion processing on one branch and statistical extraction on the other.

\section{KNOWLEDGE FORMALIZATION}

In this section, belief function formalism is described and the mechanisms used to formalize these two types of knowledge are discussed.

\section{A. Belief Functions, Transferable Belief Model (TBM)}

The TBM is an adaptation by Smets [15] of the belief functions' theory developed by Shafer [16] to provide a general framework to represent uncertainty. This generic knowledge representation and combination formalism allows the modeling and processing of imperfect knowledge resulting from multiple sources. 


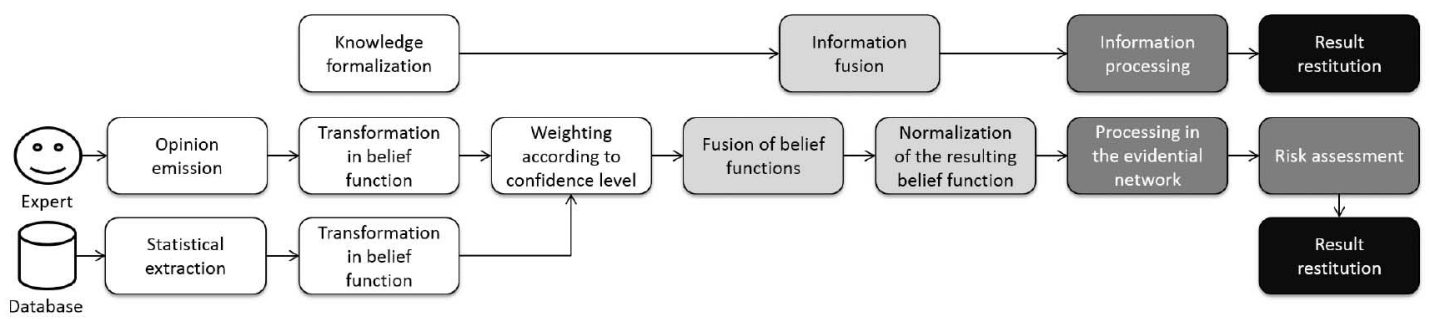

Fig. 3. Synoptic view of approach to assess risk by using directed evidential networks.

The TBM interprets a belief function as the representation of a rational agent opinion based on the belief or knowledge state of this agent, even if it is inaccurate or incomplete. The TBM is based on the assumption that reasoning under uncertainty (credal level) and decision-making (pignistic level) are two cognitive tasks of a different nature.

To model a problem using belief functions, the value of the variable $\omega$, which represents the system state, must be determined. The frame of discernment represents all possible $n$ values (or hypothesis) for the variable $\omega$ and is denoted by $\Omega=\left\{\omega_{1}, \omega_{2}, \ldots, \omega_{n}\right\}$.

The definition of the belief mass function, denoted $m^{\Omega}$, allows the translation of an observation (or advice) provided by an agent on the "power set" of $\Omega$ (denoted $\left.2^{\Omega}=\left\{\emptyset,\left\{\omega_{1}\right\},\left\{\omega_{2}\right\},\left\{\omega_{1}, \omega_{2}\right\}, \ldots,\left\{\omega_{1}, \ldots, \omega_{n}\right\}\right\}\right)$.

The power set consists of all subsets of $\Omega$. The belief mass function is defined as follows:

$$
\begin{aligned}
m^{\Omega}: 2^{\Omega} & \rightarrow \quad[0,1] \\
A \subseteq \Omega & \mapsto m^{\Omega}(A) \in[0,1] .
\end{aligned}
$$

The basic belief assignment (BBA) is the set of belief masses on the proposal $A \subseteq \Omega$ that satisfies the following equation:

$$
\sum_{A \subseteq \Omega} m^{\Omega}(A)=1 .
$$

A BBA can be transformed to highlight information and to ease the dynamic aspects of TBM, including the fusion rules. The most commonly used transformations are presented here.

1) The credibility or belief (denoted bel) symbolizes the minimum belief in proposal $A$

$$
\operatorname{bel}^{\Omega}(A)=\sum_{\emptyset \neq B \subseteq A} m^{\Omega}(B) \forall A \subseteq \Omega .
$$

2) The plausibility (denoted $\mathrm{pl}$ ) represents the maximum belief in proposal $A$

$$
\mathrm{pl}^{\Omega}(A)=\sum_{B \cap A \neq \emptyset} m^{\Omega}(B) \forall A \subseteq \Omega .
$$

As stated previously, the TBM has two distinct cognitive levels of reasoning. The transformation from credal to pignistic level is termed pignistic transformation [15]. This nonreversible transformation aims to reduce the BBA in a probability distribution so as to be compatible with decision theory. This probability distribution, denoted BetP, can be obtained from the following equation:

$$
\operatorname{BetP}\left\{m^{\Omega}\right\}\left(\omega_{i}\right)=\frac{\sum_{A \subseteq \Omega, \omega_{i} \in A} \frac{m^{\Omega}(A)}{|A|}}{\left(1-m^{\Omega}(\emptyset)\right)} \forall \omega_{i} \in \Omega .
$$

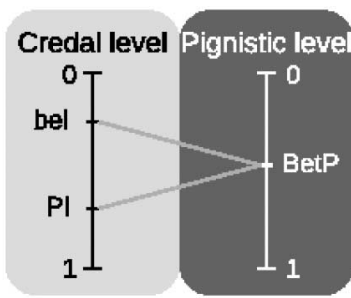

Fig. 4. Connections between credal and pignistic levels.

Fig. 4 shows the connections between the credal and pignistic levels via the notions of credibility, plausibility, and pignistic probability.

\section{B. Expert Opinion}

Usually, experts express their opinions using subjective probabilities [17]. Subjective probabilities are a simple way to model a person's belief in a hypothesis. An expert gives his opinion according to the frame of discernment $\Omega=$ $\left\{\omega_{1}, \omega_{2}, \ldots, \omega_{n}\right\}$ which is exhaustive and contains discrete and independent values.

These probability distributions are then transformed using the inverse pignistic transformation [18]. The objective of the inverse pignistic transformation is to determine, from a pignistic probability distribution $\mathrm{Bet}^{\Omega}$, the least committed BBA, which respects the least commitment principle [19].

This transformation requires the singletons of $\Omega$ to be ordered such that

$$
\begin{aligned}
\operatorname{BetP}^{\Omega}\left(\omega_{1}\right) & \geq \operatorname{BetP}^{\Omega}\left(\omega_{2}\right) \geq \cdots \geq \operatorname{BetP}^{\Omega}\left(\omega_{i}\right) \\
& \geq \cdots \geq \operatorname{BetP}^{\Omega}\left(\omega_{n}\right) \\
\text { with } n & =|\Omega| .
\end{aligned}
$$

The least committed BBA, composed of nested focal elements $A=\left\{\omega_{1}, \omega_{2}, \ldots, \omega_{i}\right\}$, can be obtained using the inverse pignistic transformation such that

$$
\begin{gathered}
\hat{m}^{\Omega}(A)=|A| \times\left(\operatorname{BetP}^{\Omega}\left(\omega_{i}\right)-\operatorname{BetP}^{\Omega}\left(\omega_{i+1}\right)\right) \\
\text { with } \operatorname{BetP}^{\Omega}\left(\omega_{n+1}\right)=0 \text { and } A=\left\{\omega_{1}, \omega_{2}, \ldots, \omega_{i}\right\} .
\end{gathered}
$$

Based on the opinion of the expert formalized as a BBA, information about the source reliability can be integrated through a mechanism termed "discounting" [16]. The goal of discounting is to weight all BBA elements by a factor $(1-\beta)$ termed reliability. The discounting rate, denoted $\beta$, is defined on the interval $[0,1]$. To respect the least commitment principle [19], the remaining mass after weighting is assigned to 
$\Omega$, which represents the total uncertainty. This mechanism adds some ignorance to the BBA with respect to the reliability of the source. Application of the discounting rate, according to (2), corresponds to the following formula:

$$
\begin{aligned}
& { }^{\beta} m^{\Omega}(A)=(1-\beta) \cdot m^{\Omega}(A) \forall A \subset \Omega \\
& { }^{\beta} m^{\Omega}(\Omega)=(1-\beta) \cdot m^{\Omega}(\Omega)+\beta .
\end{aligned}
$$

\section{Statistical Opinion}

Statistical knowledge results from a database that contains all the information capitalized during past operations. The extracted knowledge is equivalent to an "opinion" expressed on the frame of discernment, $\Omega=\left\{\omega_{1}, \omega_{2}, \ldots, \omega_{n}\right\}$.

The statistical database can be summarized as a table that contains all past cases. It synthesizes all the observations regarding the simultaneous occurrence of model events (causes, consequences, and defects). Statistical knowledge extraction involves counting the number of cases that satisfies the conditions of each variable and storing this number in a table. Th tables that contain the results of the statistical extraction must then be transformed into BBA to be incorporated into the model. To take into account knowledge induced by statistical laws, Denoeux [20] proposed the predictive belief function formalism. It allows a BBA that represents future occurrences of $X$ to be built from a number $n$ of observations of a random variable $X$.

A representative sample $\left\{X_{1}, \ldots, X_{n}\right\}$ is taken from a random variable $X$ with an unknown probability distribution, denoted $\mathbb{P}_{X}$. The objective is to build a BBA, denoted $m^{\Omega}(. ; X)$, that is less committed (within the meaning of the least commitment principle [19]) than $\mathbb{P}_{X}$ in $100 \times(1-\alpha) \%$ of cases. The coefficient $\alpha$ allows us to specify the confidence interval in the result. The predictive belief functions are based on two conditions.

1) If $\mathbb{P}_{X}$ is known, the credibility associated with $m^{\Omega}(. ; X)$ should be equal to $\mathbb{P}_{X}\left(\right.$ bel $\left.^{\Omega}(. ; X)=\mathbb{P}_{X}\right)$. However, this constraint is infeasible and must be simplified by relaxing some constraints, which eventually yields the following expression:

$$
\forall A \subset \Omega, \quad \operatorname{bel}^{\Omega}(A ; X) \stackrel{\text { Prob }}{\rightarrow} \mathbb{P}_{X}(A), \text { for } n \rightarrow \infty .
$$

2) If $n$ is finite, then bel ${ }^{\Omega}(. ; X)$ should be less committed than $\mathbb{P}_{X}$ most of the time, i.e., in $100 \times(1-\alpha) \%$ of the cases. Thus, it follows:

$$
\operatorname{Prob}\left(\operatorname{bel}^{\Omega}(A ; X) \leq \mathbb{P}_{X}(A) \forall A \subset \Omega\right) \geq 1-\alpha .
$$

When $X$ is a discrete variable on a frame of discernment $\Omega=\left\{\omega_{1}, \ldots, \omega_{K}\right\}$, as in this study, a solution can be found using simultaneous confidence intervals on the probabilities $p_{k}=\mathbb{P}\left(X=\omega_{k}\right)$. By definition, the intervals $\left[P_{k}^{-}, P_{k}^{+}\right], k=$ $1, \ldots, K$ are simultaneous confidence intervals with a confidence level of $1-\alpha$ if they satisfy the following constraint:

$$
\mathbb{P}\left(P_{k}^{-} \leq p_{k} \leq P_{k}^{+}, k=1, \ldots, K\right) \geq 1-\alpha .
$$

The choice of confidence level $1-\alpha$ allows a compromise between confidence interval size and the probability that the real probability value is in this interval. The asymptotic simultaneous confidence intervals proposed by Goodman [21] are obtained by $\mathrm{Chi}^{2}$ approximation of the polynomial distribution:

$$
\begin{aligned}
& P_{k}^{-}=\frac{b+2 N_{k}-\sqrt{\Delta_{k}}}{2(n+b)} \\
& P_{k}^{+}=\frac{b+2 N_{k}+\sqrt{\Delta_{k}}}{2(n+b)}
\end{aligned}
$$

where $n$ is the total number of observations, $N_{k}$ is the number of observations in the category $\omega_{k}, b=\chi_{1-\frac{\alpha}{K}}^{2}(1)$ is the fractile of the $\mathrm{Chi}^{2}$ law at the level $1-\frac{\alpha}{K}$ with a degree of freedom of 1 , and $\Delta_{k}=b\left(b+\frac{4 N_{k}\left(n-N_{k}\right)}{n}\right)$.

The BBA construction from this simultaneous confidence interval differs according to the number of hypotheses $K$. Therefore, the following holds depending on the cardinality of the frame of discernment.

1) If $K=2$, then $P^{-}$is a belief function

$$
\begin{aligned}
& m^{\Omega}\left(\left\{\omega_{1}\right\}\right)=P_{1}^{-} \\
& m^{\Omega}\left(\left\{\omega_{2}\right\}\right)=P_{2}^{-} \\
& m^{\Omega}(\{\Omega\})=1-P_{1}^{-}-P_{2}^{-} .
\end{aligned}
$$

2) If $K=3$, then $P^{-}$is also a belief function

$$
\begin{aligned}
& m^{\Omega}\left(\left\{\omega_{k}\right\}\right)=P_{k}^{-}, \quad k=1,2,3 \\
& m^{\Omega}\left(\left\{\omega_{1}, \omega_{2}\right\}\right)=1-P_{3}^{+}-P_{1}^{-}-P_{2}^{-} \\
& m^{\Omega}\left(\left\{\omega_{1}, \omega_{3}\right\}\right)=1-P_{2}^{+}-P_{1}^{-}-P_{3}^{-} \\
& m^{\Omega}\left(\left\{\omega_{2}, \omega_{3}\right\}\right)=1-P_{1}^{+}-P_{2}^{-}-P_{3}^{-} \\
& m^{\Omega}(\{\Omega\})=\frac{b}{n+b} .
\end{aligned}
$$

3) If $K>3$, then $P^{-}$is generally not a belief function because it does not necessarily satisfy the formal definition of a belief function [20]. Since this situation was not necessary in this study, this idea will not be developed further.

\section{FUSION OF OPINIONS}

Belief function formalism provides various rules that allow the fusion of several opinions resulting from heterogeneous sources. The two most common rules are the conjunctive rule of combination (CRC), denoted $@$, which allows the fusion of BBA from distinct and reliable sources and the disjunctive rule of combination (DRC), denoted (S), which allows the fusion of BBA from distinct sources when at least one of the sources is reliable. The $\mathrm{CRC}$ formula is presented as

$$
\left(m_{1}^{\Omega} @ m_{2}^{\Omega}\right)(A)=\sum_{B \cap C=A} m_{1}^{\Omega}(B) \cdot m_{2}^{\Omega}(C) \forall A, B, C \subseteq \Omega .
$$

The DRC formula is presented as

$$
\left(m_{1}^{\Omega}\left(m_{2}^{\Omega}\right)(A)=\sum_{B \cup C=A} m_{1}^{\Omega}(B) \cdot m_{2}^{\Omega}(C) \forall A \subseteq \Omega .\right.
$$

In this study, the CRC or DRC methods could not be used, because the sources are not independent (nondistinct) since different experts share the same information ground to elaborate 
their opinions. Since the sources are considered reliable-the source reliability is already taken into account during knowledge formalization via the use of discounting and predictive belief functions- the choice was made to use the cautious conjunctive rule of combination (CCRC) to merge expert opinions with the database. This approach allows knowledge from reliable but nondistinct sources. The CCRC, proposed by Denoeux [22], uses the canonical decomposition introduced by Shafer [16]. The canonical decomposition consists of expressing a nondogmatic BBA (nondogmatic means $m^{\Omega}(\{\Omega\})>0$ ) using a conjunctive combination of simple BBA, denoted $A^{w(A)}$. Since $A^{w(A)}$ is a simple BBA, it only contains two focal elements: $m^{\Omega}(A)=1-w(A)$ and $m^{\Omega}(\Omega)=w(A)$. Smets [23] defined the notion of a simple generalized BBA (GSBBA) to define a BBA canonical decomposition. A GSBBA is defined by a function $\mu: 2^{\Omega} \rightarrow \Re$ such that

$$
\begin{aligned}
& \mu(A)=1-w \\
& \mu(\Omega)=w \\
& \mu(B)=0, \quad \forall B \in 2^{\Omega} \backslash\{A, \Omega\} \\
& \text { with } B \neq \Omega \text { and where } w \in(0,+\infty) .
\end{aligned}
$$

The CCRC uses the notion of GSBBA and the "minimum" t-norm operator, denoted $\wedge$, to combine distributions. The CCRC, denoted $₫$, can be written as follows:

$$
\begin{aligned}
& w_{1 \bigotimes_{2}}^{\Omega}(A)=\left(w_{1}^{\Omega} @ w_{2}^{\Omega}\right)(A)=w_{1}^{\Omega}(A) \wedge w_{2}^{\Omega}(A) \\
& m_{1 \bigotimes_{2}}^{\Omega}(A)=\left(m_{1}^{\Omega} @ m_{2}^{\Omega}\right)(A)=1-w_{1 \bigotimes_{2}}^{\Omega}(A)
\end{aligned}
$$

Note that the CCRC generates a mass assigned to the empty set $\left(m^{\Omega}(\emptyset) \neq 0\right)$. Information on the empty set can be very useful because it represents conflict between the information sources [24]. However, when the number of experts grows, the number of uses of the combination rule and therefore the mass attributed to the conflict increases. Therefore, the BBA resulting from the fusion before processing the directed evidential network must be normalized [24] to prevent the conflict from becoming the predominant information. This phenomenon reduces the influence of other information in the directed evidential network and justifies the use of the normalized CCRC

$$
\left(m_{1} \bigotimes{ }^{*} m_{2}\right)(A)=\left\{\begin{array}{lr}
\frac{\left(m_{1} \bigotimes m_{2}\right)(A)}{1-\left(m_{1} \bigotimes m_{2}\right)(\emptyset)} & \forall A \subseteq \Omega \text { if } A \neq \emptyset \\
0 & \text { if } A=\emptyset .
\end{array}\right.
$$

\section{Processing With Directed Evidential Networks}

After presenting the knowledge formalization and the methods to combine it, the processing of this knowledge to achieve risk assessment will be discussed. As mentioned earlier, this study aims to extend the risk assessment process developed with Bayesian networks into an evidential framework based on belief functions. To this end, directed evidential networks, introduced by Yaghlane [25], are used. This formalism is similar to Bayesian networks [2] but uses conditional belief functions instead of conditional probabilities. Each edge of the network represents a conditional relationship between two variables, represented by nodes. Each variable is defined on a frame of discernment that represents the set of possible values. Nodes without a parent are characterized by a priori belief functions, and nodes with parents are characterized by conditional belief functions (i.e., BBA conditionally to the values of their parents).

The generalized bayes theorem (GBT) and the DRC are used to infer and propagate knowledge in the network. The Bayes theorem is a probabilistic inference mechanism used in Bayesian networks. It can bind a series of hypotheses, characterized by occurrence probabilities, with a series of observations that represent the actual state of the system. The GBT [26] is the generalization of this mechanism to the TBM.

The hypothesis space is termed $\Theta=\left\{\theta_{1}, \theta_{2}, \ldots, \theta_{i}\right\}$ and the observation space is termed $\Omega=\left\{\omega_{1}, \omega_{2}, \ldots, \omega_{k}\right\}$. To represent links between observations and hypotheses, notation related to conditional belief functions is used $\left(p l^{\Omega}[\theta]\right.$ is the plausibility on $\Omega$ conditioned by $\theta$, where [] symbolizes conditioning). A directed evidential network is composed of nodes and edges. An edge represents the causal relationship between nodes. From the perspective of the variables, which are represented by nodes, the edge indicates that the node $\Omega$, which represents the observation space, is conditioned by the node $\Theta$, which represents the hypothesis space. Therefore, the plausibility distribution on $\Omega$ is $p l^{\Omega}[\theta]$.

The node $\Theta$ is characterized by an a priori BBA, $m_{0}^{\Theta}$, and the node $\Omega$ is characterized by a conditional BBA, $m^{\Omega}[\theta]$. Knowledge is then propagated according to the edge direction if the node $\Theta$ receives a new distribution of masses, $m_{0}^{\Theta}$. Therefore, the node $\Omega$ is updated with respect to this new information. This propagation type, termed "forward propagation," allows the calculation of the plausibility distribution $p l^{\Omega}$, using the GBT and the DRC by taking into account the inclusion of $a$ priori knowledge

$$
\begin{aligned}
\mathrm{pl}^{\Omega}(\omega) & =\sum_{\theta \subseteq \Theta} m_{0}^{\Theta}(\theta) \times p l^{\Omega}[\theta](\omega) \quad \forall \omega \subseteq \Omega \\
\mathrm{pl}^{\Omega}[\theta](\omega) & =1-\prod_{\theta_{i} \in \Theta}\left(1-p l^{\Omega}\left[\theta_{i}\right](\omega)\right) .
\end{aligned}
$$

Knowledge can also be propagated in the opposite direction of the edge if the node $\Omega$ receives a new BBA, $m^{\Omega}$. The node $\Theta$ is then updated to reflect this new information. This step, termed "backward propagation," allows for the calculation of the plausibility distribution $p l^{\ominus}$, using the following equation, which is based on the GBT, to determine the plausibility $p l^{\Theta}\left[\omega_{i}\right]$ :

$$
\begin{aligned}
\mathrm{pl}^{\Theta}(\theta)= & \sum_{\omega \subseteq \Omega} m^{\Omega}(\omega) \times\left(1-\prod_{\omega_{i} \in \omega}\left(1-\mathrm{pl}^{\Theta}\left[\omega_{i}\right](\theta)\right)\right) \forall \theta \subseteq \Theta \\
& \mathrm{pl}^{\Theta}[\omega](\theta)=1-\prod_{\theta_{i} \in \theta}\left(1-\mathrm{pl}^{\Omega}\left[\theta_{i}\right](\omega)\right) .
\end{aligned}
$$

If both nodes have to be updated because new knowledge has been assigned to the two nodes, knowledge must be propagated first by using forward propagation and then by using backward propagation. Finally, the results of the two propagation cycles are merged using the CRC. 


\section{RESULTING INDICATORS}

The main drawback of belief functions is that a user who does not have the required background knowledge cannot easily interpret them. The introduction of indicators for decisionmakers is very important. Two indicators are introduced to ensure maximum accuracy. A risk indicator is proposed and an uncertainty indicator is developed to balance the information conveyed by the risk indicator according to the data quality.

\section{A. Risk Indicator}

The chosen solution to assess risk is to use the credibility and plausibility distributions to calculate minimum and maximum risks. At this stage, the introduction of the notion of compatible probabilities is important to achieve this purpose. If a probability distribution $\mathcal{P}$ and a BBA, $m$, represented by credibility (bel) and plausibility (pl) distributions represent the occurrence of the same event, they are said to be compatible if and only if

$$
\operatorname{bel}(A) \leq \mathcal{P}(A) \leq \operatorname{pl}(A)
$$

In this case, this constraint is considered verified. Two measures can be deduced: the minimum risk $\underline{\mathcal{R}}$, and the maximum risk $\overline{\mathcal{R}}$. These measures are determined using a utility function, which represents impact levels because of consequence events in a system. It is expressed as a value on a scale of 0 to 100 according to the consequence state (the sum of the impacts of different consequence states must be less than or equal to 100). The minimum risk is calculated by multiplying the credibility and impact for each singleton of the frame of discernment, followed by the addition of these results expressed on each singleton. The maximum risk exploits the same formula but uses plausibility instead of credibility. The formulas determining these measures for a BBA, $m^{\Omega}$, with a credibility bel ${ }^{\Omega}$, a plausibility $\mathrm{pl}^{\Omega}$, and an impact $I^{\Omega}$ are

$$
\begin{aligned}
& \underline{\mathcal{R}}^{\Omega}=\sum_{\omega_{i} \in \Omega} I^{\Omega}\left(\omega_{i}\right) \times \operatorname{bel}^{\Omega}\left(\omega_{i}\right) \\
& \overline{\mathcal{R}}^{\Omega}=\sum_{\omega_{i} \in \Omega} I^{\Omega}\left(\omega_{i}\right) \times \mathrm{pl}^{\Omega}\left(\omega_{i}\right) .
\end{aligned}
$$

With this method, the decision-maker can adopt different strategies to compare the results of optimistic (using the minimum risk) and pessimistic (using the maximum risk) approaches.

Counterbalancing the risk indicator according to the different information quality properties that are introduced in the evidential networks is very important. Providing only a risk measure, while the network addresses imperfect information, would be too restrictive and would represent an overall information loss. So, putting some information into perspective regarding the data quality that led to this result is important. Therefore, an indicator that represents the global level of uncertainty is proposed.

\section{B. Uncertainty Indicator}

To assess the uncertainty level of each model variable, the measure of nonspecificity is used [27]. It determines the BBA uncertainty rate and is assessed by the amount of information assigned to the power set subsets with a cardinality higher than one. This measure (23) weights more heavily the elements with highest cardinality. This approach characterizes a larger uncertainty because it is applied to more than one hypothesis

$$
N(m)=\sum_{\emptyset \neq A \subseteq \Omega} m(A) \times \log _{2}(|A|)
$$

with $N(m) \in\left[0, \log _{2}(|\Omega|)\right]$ and $|A|$ the proposal cardinality.

However, this measure provides a numerical result that is difficult to interpret. Therefore, we propose to use a percentage of the maximum nonspecificity, i.e., the nonspecificity of the empty BBA that represents total ignorance. From a mathematical point of view, this new measure, denoted $N_{\%}$, is expressed by the following formula:

$$
N_{\%}(m)=\frac{\sum_{\emptyset \neq A \subseteq \Omega} m(A) \times \log _{2}(|A|)}{\log _{2}(|\Omega|)} .
$$

More detailed information for the decision-maker can also be obtained by separating the partial and total uncertainty. The decision-maker can determine whether the value of nonspecificity measure reflects total ignorance or whether the knowledge used to make the decision contains useful information. Therefore, two alternative measures, denoted $N p_{\%}$ (for partial uncertainty) and $N t_{\%}$ (for total uncertainty), are proposed and determined by the following formulas:

$$
\begin{aligned}
& N p_{\%}(m)=\frac{\sum_{\emptyset \neq A \subset \Omega} m(A) \times \log _{2}(|A|)}{\log _{2}(|\Omega|)} \\
& N t_{\%}(m)=\frac{m(\Omega) \times \log _{2}(|\Omega|)}{\log _{2}(|\Omega|)}=m(\Omega) .
\end{aligned}
$$

Notably, $N p_{\%}(m)+N t_{\%}(m)=N_{\%}(m)$. The $N_{\%}$ measure indicates the uncertainty level of a BBA. An uncertainty categorization scale similar to the one used for risk can be created to allow the decision-maker to interpret the result.

\section{Application to Aircraft DeCOnstruction}

By using knowledge generated during aircraft deconstruction, a decision-support system has been established to facilitate knowledge management, especially during the plane inspection phase. A module was developed to analyze and take advantage of the knowledge generated during this inspection phase. This experience feedback tool provides the decision-maker with an opportunity to match the functioning conditions of the aircraft with different defects in critical airplane areas via statistical and expert analyses. This framework allows for the development of an application to assess risk in critical parts and areas of an airplane. This tool uses a database and a statistical analysis module that are deployed in the DIAGNOSTAT project.

The database contains the following informations: 1) the characteristics of each aircraft (type, option, ... ); 2) the living and using conditions (long-haul or national flights, maintenance, etc.); and 3) the various defects encountered during deconstruction and/or maintenance. The statistical analysis 
TABLE I

Descriptive Table of the Event Related to the Study CAse

\begin{tabular}{|c|c|c|c|}
\hline Name & Code & States & Parent(s) \\
\hline Lightning strike & C11 & Yes, No & \\
In-flight shock & C12 & Yes, No & \\
Ground shock & C13 & Yes, No & \\
Significant impact 1.1 & D11 & Yes, No & C11,C12,C13 \\
Significant impact 1.2 & D12 & Yes, No & C11,C12,C13 \\
Significant impact 1 & D1 & Yes, No & D11,D12 \\
Surface defect & P11 & Yes, No & D1 \\
Delamination & P12 & Yes, No & D1 \\
Process error & C21 & Yes, No & \\
Maintenance quality & C22 & Good, Bad & \\
Maintenance defect 2.1 & D21 & Yes, No & C21,C22 \\
Maintenance defect 2.2 & D22 & Yes, No & C21,C22 \\
Maintenance defect 2 & D2 & Yes, No & D21,D22 \\
Patch debonding & P21 & Yes, No & D2 \\
Water Sseepage & P22 & Yes, No & D2 \\
Crack initiation 3.1 & D31 & Yes, No & P12,P21 \\
Crack initiation 3.2 & D32 & Yes, No & P12,P21 \\
Crack initiation 3 & D3 & Yes, No & D31,D32 \\
Complete fracture & P31 & Yes, No & D3 \\
Disintegration & P32 & Yes, No & D3 \\
\hline
\end{tabular}

module puts information into perspective to generate usable statistics. The capabilities of this tool are enhanced by associating a risk assessment module, which, in addition to the raw statistics from the statistical analysis module, integrates some expert knowledge to assess the risk for critical areas of the aircraft.

A case-study has been developed to implement the risk assessment model, according to DIAGNOSTAT project objectives and in partnership with an expert in the field of composite aircraft spare parts. The model exhibits three defects and their respective causes and consequences for a system composed of two composite panels. Table I summarizes the modeling of the study case using the risk assessment model that is illustrated in Fig. 6. Each node in the graphical view is linked to a variable with a code described in Table I.

A scenario as applied to aircraft deconstruction was developed to illustrate how this model behaves. In this scenario, the decision-maker does not possess additional knowledge to inform the model upon aircraft arrival (he knows nothing of the past life of the unit). During the aircraft inspection operations, a surface defect is observed (P11=Yes), and previous repairs show patch debonding (P21=Yes). Using the model, the decision-maker is able to assess the risk that a defect is present in the system and to evaluate whether the part is reusable. The addition of knowledge obtained during the inspection of the plane will allow this assessment to be refined.

A prototype has been developed to integrate the mechanisms presented above and to validate this study.

\section{A. Process Flow}

As stated above, knowledge is retrieved from two separate sources: a database [Fig. 5(a)] and expert opinion capitalized using subjective probabilities [Fig. 5(b)]. The statistical knowledge, extracted from the database by conditional counting, is converted into belief functions using predictive belief functions (Section III-C). Subjective probabilities that represent the expert knowledge are transformed into belief functions

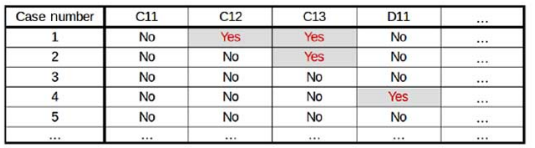

(a)

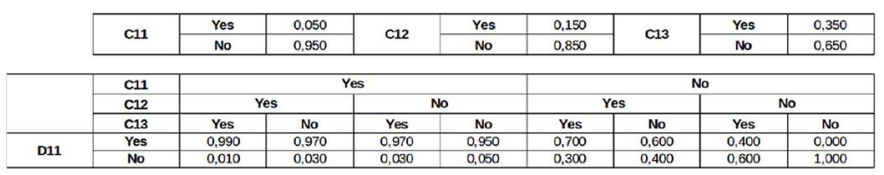

(b)

Fig. 5. Initial knowledge overview. (a) Database example. (b) Expert opinion example.

using inverse pignistic transformation (Section III-B). Once this knowledge is formalized, opinions from these two sources are merged using the Normalized CCRC (Section IV). At this point, a BBA that represents the combined knowledge is obtained for each variable in the model. These BBAs are then introduced into the directed evidential network and a first phase of processing is launched (Section V). The results at the end of this first phase are presented in Section VII-B. Finally, during inspection of the airplane, new knowledge is discovered and added to the directed evidential network by modifying the contents of the variables, and a new processing phase is launched to obtain results revised according to the additional knowledge. These results are presented in Section VII-C.

\section{B. Results Before Knowledge Addition}

Fig. 6(a) presents the results of the model before the introduction of knowledge related to the scenario. Four types of nodes exist in this modeling.

1) Chance nodes that represent belief in the occurrence of an event [i.e., node C11 in Fig. 6(a)]. These nodes contain a graphical indicator that represents belief and uncertainty. A belief in the positive result of the event ( $\mathrm{C} 11$ does not occur) is represented by the light gray part of the bar. A belief in the negative result of the event (C11 occurs) is represented by the dark gray part of the bar. The length of the bar is inversely proportional to the uncertainty.

2) Chance nodes frozen by knowledge addition represent an event that has occurred with certainty [i.e., node P11 in Fig. 6(b)].

3) Utility nodes represent the impact of a consequence [i.e., node I11 in Fig. 6(a)]. The impact value of the negative event, contained in the node, is always between 0 and 100 (see Section VI-A).

4) Decision nodes represent the risk measure, $\underline{\mathcal{R}}$ and $\overline{\mathcal{R}}$, of a consequence [i.e., node R11 in Fig. 6(a)]. These nodes contain a graphical indicator that represents minimum and maximum risk measures (see Section VI-A). The length of the bar is proportional to the risk measure.

The first observation shows that the uncertainty is very important in the chance nodes located at the bottom of the network. This uncertainty affects the risk indicator that shows a large gap between the minimum and maximum risk. The number of levels in the network is supposed to correlate positively with the degree of uncertainty created by propagation mechanisms. 


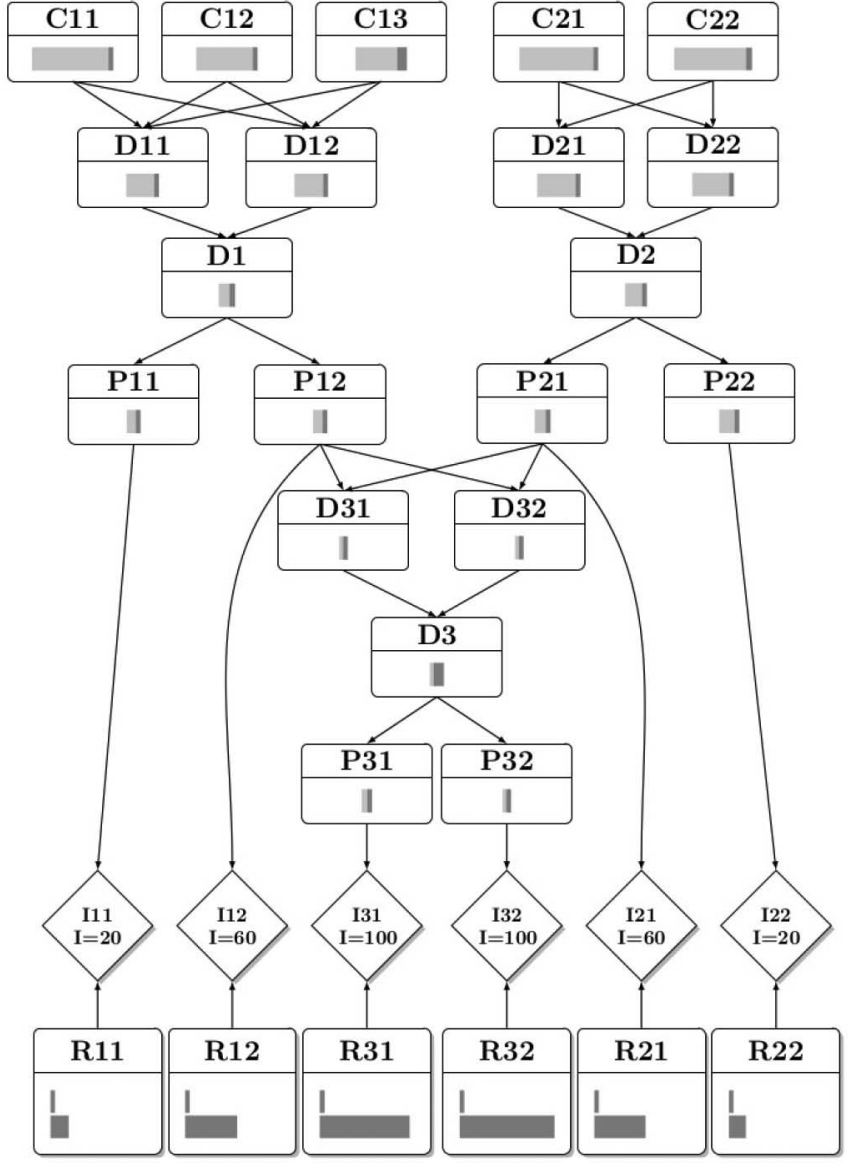

(a)

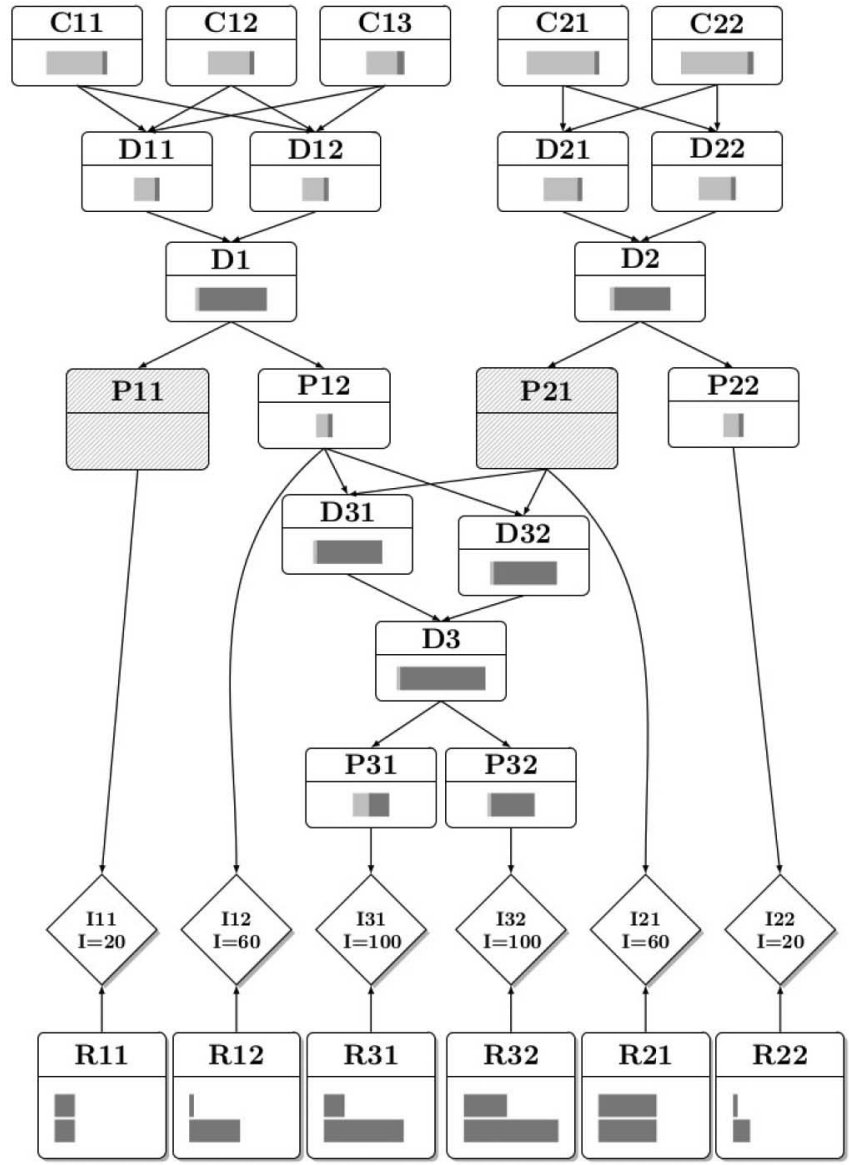

(b)

Fig. 6. Results of processing with directed evidential networks. (a) Initial processing. (b) After the addition of scenario knowledge.

\section{Results After Knowledge Addition}

The scenario begins by setting P11 and P21 nodes $\left(m^{P 11}(\{\right.$ Yes $\})=1$ and $m^{P 21}(\{$ Yes $\left.\})=1\right)$. Fig. 6(b) presents results of this scenario after adding knowledge obtained during inspection.

The consistency of the evolution of results can be verified. A significant decrease in uncertainty of the resulting network is visible. Therefore, the risk measures are more accurate. The uncertainty, and thus the gap between minimum and maximum risk measures, is reduced by increasing the minimum risk because, in this case, the added knowledge addresses the occurrence of negative events (P11 and P21). However, it should be noted that the reduction in uncertainty can also be done by adding knowledge about the nonoccurrence of a negative event, which will reduce the maximum risk. The importance of the uncertainty generated by the propagation mechanism is supposed to correlate positively with the distance (propagation steps) of the studied node from the node where knowledge is introduced.

\section{General Remarks About the Model}

These results represent a "rough" model with relatively substantial uncertainty. Indeed, the choice of belief functions and therefore directed evidential networks is related to the fact that there is a lack of sufficient data to address the problem statistically. This lack of data creates uncertainty, which affects the final results.

To illustrate the importance of uncertainty on the final result, some tests that vary the uncertainty level of the "Cause" nodes (C11 to C22) were conducted on two model states (i.e., before and after the addition of knowledge). Fig. 7 illustrates the impact of the mean uncertainty level of the "Cause" nodes on the "Consequence" nodes (P11 to P32) before the addition of knowledge. The uncertainty level on each node has been quantified through the uncertainty measure, $N_{\%}$ (Section VI-B). The uncertainty on the "Cause" nodes had a significant impact on the "Consequence" nodes. Moreover, the uncertainty on intermediate levels (nodes D) affects these "Consequence" nodes significantly. This is why the uncertainty level of the most distant "Consequence" nodes from the "Cause" nodes (P31 and P32) is always greater than $85 \%$ regardless of the uncertainty level of the "Cause" nodes.

The same tests were conducted after knowledge addition, i.e., after certain knowledge injection in the model $\left(N_{\%}^{P 11}=\right.$ $N_{\%}^{P 21}=0$ ). A significant reduction in uncertainty on the P31 and P32 nodes is observed. The same trend can be observed for other "Consequence" nodes but the variation is less important. Table II summarizes these findings.

These tests demonstrate the utility of the uncertainty measure proposed in Section VI-B to inform the decision-maker when 


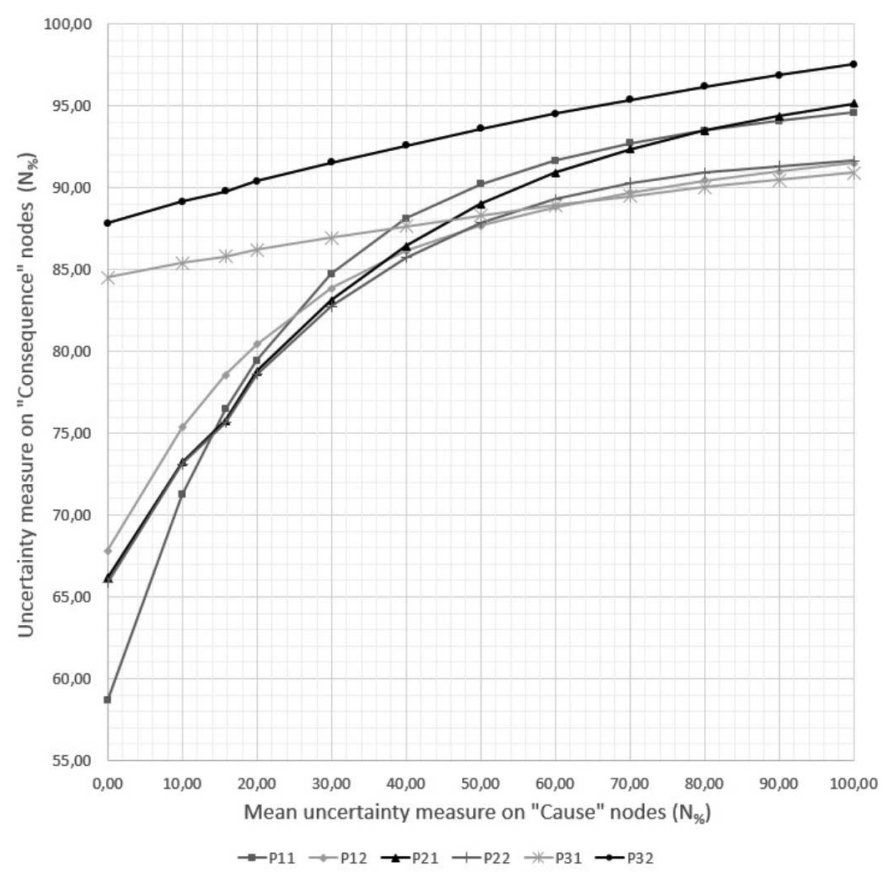

Fig. 7. Influence of "Cause" node uncertainty on the "Consequence" nodes.

TABLE II

IMPACT OF KNOWLEDGE ADDITION ON THE UNCERTAINTY LEVEL IN THE MODEL

\begin{tabular}{|c|c|c|c|c|c|c|}
\hline \multirow{2}{*}{$N_{\%}$ of "Cause" nodes } & \multicolumn{3}{|c|}{ Before knowledge addition (\%) } & \multicolumn{3}{|c|}{ After knowledge addition (\%) } \\
\hline & 0 & 50 & 100 & 0 & 50 & 100 \\
\hline P12 & 67.82 & 87.67 & 91.50 & 61.34 & 78.13 & 80.71 \\
\hline P22 & 65.86 & 89.00 & 91.64 & 65.85 & 87.85 & 91.64 \\
\hline P31 & 84.52 & 88.31 & 90.90 & 61.85 & 62.21 & 62.30 \\
\hline P32 & 87.81 & 93.51 & 97.5 & 53.46 & 54.01 & 54.14 \\
\hline
\end{tabular}

there is a need to add knowledge in the model (e.g., requesting an extensive aircraft inspection) if the uncertainty level is too great.

According to [26], the model will gradually approach its probabilistic counterpart if the amount of data increases. Thus, the result will be the same that obtained with Bayesian networks if the data set used contains only Bayesian BBA.

\section{CONCLUSION}

We have presented an approach designed to assess the risk during the deconstruction of end-of-life aircraft. This approach allows for the formalization, combination, and processing of uncertain knowledge resulting from heterogeneous information sources. The heterogeneity of information sources used in this study is related to the nature of the feedback mechanisms used in industry, i.e., cognitive and statistical experience feedbacks. Moreover, some indicators that allow the decision-maker to be informed about the risk level but also about the data quality concerning the uncertainty have been developed.

The prospects of this research work relate mainly to two issues.

First, understanding the behavior of the risk assessment model based on directed evidential networks faced with uncertainty appears to be important. As shown above, the risk assessment approach based on directed evidential networks yields results with significant uncertainty. This uncertainty arises because of uncertainty in the initial data and that generated by the propagation mechanisms. Testing this hypothesis and understanding the influence of these two factors on the final result would be interesting. A series of tests designed to explain the impact of uncertainty on the behavior of the original model and to characterize the influence of different propagation steps in a directed evidential network on the increase in uncertainty can be implemented. Furthermore, the choice of combination rule has a direct impact on the proportion of conflict and uncertainty in the results. Because the CCRC method was used to merge expert opinions and statistics and CRC and DRC mechanisms were used to instrument inference in directed evidential networks, determining the influence, in terms of uncertainty, of the choice of the combination rule on final indicator wouInfluence of "Cause" node uncertainty on the "Consequence" nodes.ld be interesting. In addition, building directed evidential networks based on CCRC to reflect that the variables of the problem represented by a directed evidential network could be considered to be nondistinct constitutes another challenge.

\section{REFERENCES}

[1] E. Villeneuve, C. Beler, F. Peres, and L. Geneste, "Hybridization of Bayesian networks and belief functions to assess risk: Application to aircraft disassembly," in Proc. Int. Conf. Ind. Eng. Syst. Manage. (IESM'11), 2011, pp. 1070-1079.

[2] F. Jensen and T. Nielsen, Bayesian Networks and Decision Graphs. New York, NY, USA: Springer, 2007.

[3] D. Dubois and H. Prade, "Formal representation of uncertainty," in Decision-Making Process, D. Bouyssou, D. Dubois, M. Pirlot and H. Prade, Eds. Hoboken, NJ, USA: Wiley, 2009.

[4] J. Nowak, S. Sarkani, and T. Mazzuchi, "Risk assessment for a national renewable energy target-Part II: Employing the model," IEEE Syst. J., vol. PP, no. 99, pp. 1-12, Jan. 2014.

[5] J. Botero, C. Beler, D. Noyes, and L. Geneste, "Integration of experience feedback into the product lifecycle: An approach to best respond to the bidding process," in Proc. 14th IFAC Symp. Inf. Control Prob. Manuf., 2012, pp. 1095-1100.

[6] A. Villemeur, Reliability, Availability, Maintainability and Safety Assessment. Hoboken, NJ, USA: Wiley, 1992.

[7] Committee of Sponsoring Organizations of the Treadway Commission, Enterprise Risk Management-Integrated Framework, 2004.

[8] Y. Choi, X. Ye, L. Zhao, and A. Luo, "Optimizing enterprise risk management: A literature review and critical analysis of the work of WU and OLSON," Ann. Oper. Res., pp. 1-20, Feb. 2015.

[9] R. Walker, Winning With Risk Management. Singapore: World Scientific, 2013.

[10] E. O'Donnell, "Enterprise risk management: A systems-thinking framework for the event identification phase," Int. J. Account. Inf. Syst., vol. 6, no. 3, pp. 177-195, 2005.

[11] D. D. Wu, X. Kefan, C. Gang, and G. Ping, "A risk analysis model in concurrent engineering product development," Risk Anal., vol. 30, no. 9, pp. 1440-1453, 2010.

[12] D. D. Wu and D. L. Olson, "Enterprise risk management: A DEA VAR approach in vendor selection," Int. J. Prod. Res., vol. 48, no. 16, pp. 49194932, 2010.

[13] D. D. Wu and D. L. Olson, "Enterprise risk management: Small business scorecard analysis," Prod. Planning Control, vol. 20, no. 4, pp. 362-369, 2009.

[14] D. D. Wu and D. L. Olson, "Enterprise risk management: Coping with model risk in a large bank," J. Oper. Res. Soc., vol. 61, no. 2, pp. 179-190, 2010.

[15] P. Smets and R. Kennes, "The transferable belief model," Artif. Intell., vol. 66, pp. 191-234, 1994.

[16] G. Shafer, A Mathematical Theory of Evidence. Princeton, NJ, USA: Princeton Univ. Press, 1976.

[17] L. Savage, The Foundations of Statistics. Hoboken, NJ, USA: Wiley, 1954. 
[18] P. Smets, "Quantified epistemic possibility theory seen as an hyper cautious transferable belief model," Rencontres Francophones sur les Logiques Floues et ses Applications, 2000, pp. 343-353.

[19] Y. Hsia, "Characterizing belief with minimum commitment," in Proc. Int. Joint Conf. Artif. Intell. (IJCAI'91), 1991, vol. 2, pp. 1184-1189.

[20] T. Denoeux, "Construction of predictive belief functions using a frequentist approach," in Proc. Conf. Inf. Process. Manage. Uncertainty (IPMU), 2006, vol. 2, pp. 1412-1419.

[21] L. Goodman, "On simultaneous confidence intervals for multinomial proportions," Technometrics, vol. 7, no. 2, pp. 247-254, 1965.

[22] T. Denoeux, "Conjunctive and disjunctive combination of belief functions induced by nondistinct bodies of evidence," Artif. Intell., vol. 172, pp. 234-264, 2008.

[23] P. Smets, "The canonical decomposition of a weighted belief," in Proc. 14th Int. Joint Conf. Artif. Intell., San Francisco, CA, USA, 1995, vol. 2, pp. 1896-1901.

[24] E. Lefevre, O. Colot, and P. Vannoorenberghe, "Belief function combination and conflict management," Inf. Fusion, vol. 3, pp. 149-162, 2002.

[25] B. Ben Yaghlane, P. Smets, and K. Mellouli, Directed Evidential Networks With Conditional Belief Functions. New York, NY, USA: Springer, 2004, pp. 291-305.

[26] P. Smets, "Belief functions: The disjunctive rule of combination and the generalized Bayesian theorem," Int. J. Approximate Reasoning, vol. 9, pp. $1-35,1993$.

[27] D. Dubois and H. Prade, "The principle of minimum specificity as a basis for evidential reasoning," in Uncertainty in Knowledge-Based Systems, B. Bouchon and R. Yager, Eds. New York, NY, USA: Springer, 1987, pp. $75-84$.

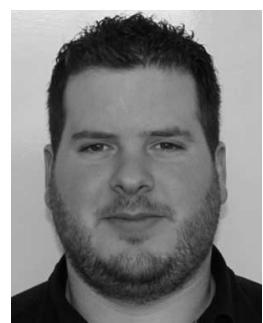

Éric Villeneuve received the Engineering degree in industrial engineering from the National Engineering School of Tarbes (ENIT - INPT), Tarbes, France, in 2009 and the Ph.D. degree in decision-support systems from the National Polytechnic Institute of Toulouse (INPT), Toulouse, France, in 2012.

$\mathrm{He}$ is a Researcher with the Production Management Laboratory (LGP), National Engineering School of Tarbes, National Polytechnic Institute of Toulouse (ENIT-INPT), Tarbes, France. His research interests include knowledge management, experience feedback, risk management, and uncertain knowledge processing.

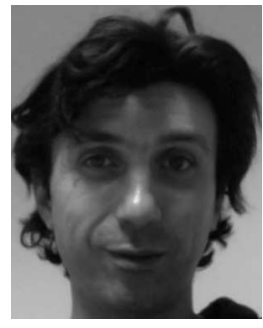

Cédrick Béler received the $\mathrm{Ph} . \mathrm{D}$. degree in industrial systems from the University Paul Sabatier, Toulouse, France, in 2008.

$\mathrm{He}$ is an Associate Professor with the National Engineering School of Tarbes, National Polytechnic Institute of Toulouse (ENIT-INPT), Tarbes, France, since 2010. He is currently a Member of the "Cognitive and Decisional Systems" of the Production Management Laboratory (LGP-SDC), Tarbes, France. His research interests include knowledge engineering, knowledge representation, and inference while considering uncertainty and risks.

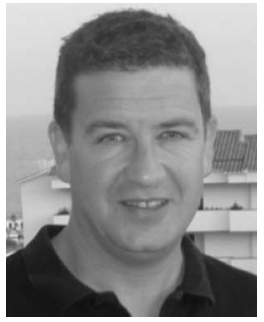

François Pérès received the Ph.D. degree in mechanical and industrial engineering from the University of Bordeaux, Talence, France, in 1996.

$\mathrm{He}$ is a French Engineer with the University of Bordeaux. He joined the Ecole Centrale of Paris, Châtenay-Malabry, France, as an Assistant Professor. $\mathrm{He}$ is currently a Full Professor with the Toulouse Midi-Pyrénées Federal University, Toulouse, France, and also with the Laboratoire Génie de Production, Tarbes, France, as a Leader of the Uncertainty, Risk, and Decision Group. His research interests include risk management applied to the design and maintenance strategies of manufactured products as well as human factor-based risk assessment approach.

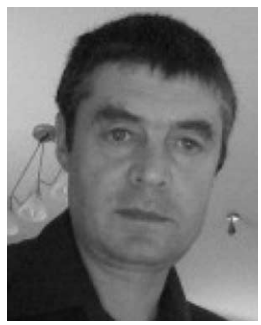

Laurent Geneste received the Ph.D. degree in industrial engineering from the University Paul Sabatier, Toulouse, France, in 1995, and accreditation to supervise research in 2002.

$\mathrm{He}$ is a Professor with the National Engineering School of Tarbes, National Polytechnic Institute of Toulouse (ENIT-INPT), Toulouse, France. He is currently a Head of the "Cognitive and Decisional Systems" of the Production Management Laboratory, Tarbes, France. His research interests include knowledge engineering and more specifically to experience feedback for problem solving in industrial organizations.

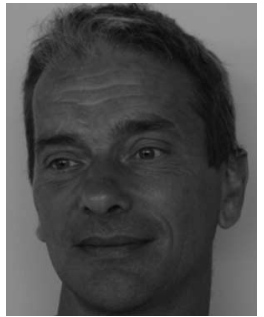

Éric Reubrez received the Engineering degree from the National Engineer School of Metz (ENIM), Metz, France.

Since 1990, he has been with the National Engineer School of Tarbes (ENIT-INPT), Tarbes, France. After working eight years in the relationships between school and industry, he is currently assigned to the Technology Transfer Center of Production Management Laboratory (LGP-ENIT). His activity is to develop computer applications using web technologies, to exploit and enhance research results, primarily in the areas of experience feedback and decision-support systems. 